ISSN 1991-8631

Review Paper

http://indexmedicus.afro.who.int

\title{
Polymorphismes du gène $A B C B 1$ (MDR1) et impacts fonctionnels dans le transport de xénobiotiques - Particularités chez les sujets Noirs Subsahariens
}

\author{
Aurel Constant ALLABI ${ }^{1 *}$, Anatole LALEYE ${ }^{2}$, Olaréwadjou AMOGOU ${ }^{3}$, \\ Abiola AGONKOUN ${ }^{3}$, Jean-Luc GALA ${ }^{4}$ et Yves HORSMANS ${ }^{5}$ \\ ${ }^{1}$ Unité de Pharmacologie, Faculté des Science de la Santé de l'Université d'Abomey-Calavi, Benin. \\ ${ }^{2}$ Laboratoire de Cytogénétique, Faculté des Science de la Santé de l'Université d'Abomey-Calavi, Benin. \\ ${ }^{3}$ Faculté des Sciences et Techniques de l'Université d'Abomey-Calavi, Benin. \\ ${ }^{4}$ Centre des Technologies Moléculaires Appliquées, Université Catholique de Louvain, Bruxelles, Belgique. \\ ${ }^{5}$ Unité de Pharmacologie clinique, Cliniques Universitaires Saint-Luc, Bruxelles, Belgique. \\ *Auteur correspondant, E-mail : acallabi@hotmail.com; Tel : +22995734900
}

\section{RESUME}

Les différences interindividuelles observées dans la réponse aux médicaments sont en partie dues aux polymorphismes du gène $A B C B 1$ codant pour la glycoprotéine- $\mathrm{P}$ ( $\mathrm{P}$-gp), une protéine de transport des médicaments. Cette revue a pour but de faire le point des connaissances sur les polymorphismes du gène $A B C B 1$, leur relevance fonctionnelle et clinique et de préciser les particularités au sein de la population noire subsharienne. Le rôle du gène $(A B C B 1) M D R 1$ dans la variabilité pharmacocinétique ou pharmacodynamique a été démontrée clairement in vitro, dans des modèles animaux et lors d'études cliniques. Le polymorphisme C3435T est associé à une diminution de l'expression protéique de la $\mathrm{P}$-gp et, par voie de conséquence, de son activité. L'existence d'une forte association entre les polymorphismes C3435T et G2677T a suggéré alors que la différence fonctionnelle observée peut être attribuée également au deuxième polymorphisme i.e le G2677T (exon 21). Il a été identifié chez les noirs africains de nouveaux polymorphismes et des haplotypes particuliers. Différnts travaux ont démontré également que le gène $A B C B 1$ intervient dans le suivi thérapeutique des patients mis sous immunosuppresseurs, anticancéreux, antidépresseurs, antirétroviraux ou antihypertenseurs. Des perspectives fort intéressantes en pharmacothérapie avec entre autres l'utilisation de modulateurs de la P-gp sont d'actualité.

(C) 2012 International Formulae Group. All rights reserved.

Mots clés : Pharmacogénétique, glycoprotéine-P, médicaments, substances exogènes, Noirs Subsahariens

\section{INTRODUCTION}

La réponse aux médicaments est extrêmement variable d'un individu à l'autre. $\mathrm{La}$ variabilité de cette réponse, souvent difficile à prévoir, est une limitation importante à l'utilisation des médicaments. De plus, les médicaments peuvent induire des effets indésirables nocifs responsables de plus de 100 000 décès par an aux Etats-Unis, les classant au quatrième rang des causes de mortalité, et représentent un coût annuel global (hospitalisations, arrêts de travail) de 100 milliards de \$US (Lazarou et al., 1998). Chaque année, en France, les effets indésirables liés aux 
médicaments seraient responsables d'environ 128000 hospitalisations, pour un coût global estimé à 320 millions d'euros (extrait d'un rapport de l'Agence française de sécurité sanitaire des produits de santé, 2001). Une enquête réalisée par le réseau des Centres régionaux de pharmacovigilance en 1998 a montré que l'incidence des hospitalisations liée aux effets indésirables des médicaments était de $3,2 \%$ en France (Pouyanne et al., 2000). Les effets indésirables liés aux médicaments constituent donc un problème médical et de santé publique important.

En dehors d'erreurs d'indications, de posologie ou d'utilisation, qui participent pour une large part à l'inefficacité et à la toxicité des médicaments, les causes de la variabilité de réponse aux traitements médicamenteux peuvent être d'origine :

- physiologique ou pathologique: âge, sexe, grossesse, sévérité de la maladie, pathologies associées ;

- environnementale: alimentation, coadministration de médicaments, tabagisme etc..

- génétique: variations génétiques du métabolisme et du transport des médicaments, des cibles pharmacologiques (récepteurs). C'est là précisément qu'intervient la pharmacogénétique pour étudier les mécanismes d'origine génétique intervenant dans la variabilité interindividuelle de la réponse aux médicaments. Elle doit permettre à terme d'identifier les individus ayant une réponse 'anormale'. La survenue de crises d'anémies hémolytiques après prise de médicaments « oxydants » comme la primaquine (antipaludique) chez les individus déficitaires en glucose-6-phosphate déshydrogénase (Burgoine et al., 2010; Beutler, 1993), l'apparition de neuropathies périphériques suite à l'administration de l'isoniazide chez certains sujets (Fekih et al., 2011) ou la survenue d'apnées après anesthésie à la succinylcholine chez certains patients ( Pantuck, 1993; Sujatha et al., 2011), représentent les premiers exemples célèbres illustrant le rôle des différences génétiques dans la variation interindividuelle de la réponse aux médicaments. Depuis lors de très nombreux exemples d'anomalies génétiques modifiant la réponse aux médicaments ont été rapportés (Ingelman-Sundberg et al., 1999 ; Daly and King, 2003); Oscarson, 2003), et la nécessité de prévoir, et surtout de prévenir, cette modification est devenue évidente.

Les polymorphismes du gène $A B C B 1$ chez les sujets de race noire subsaharienne constituent un bon exemple d'évolution des connaissances dans l'explication de la variation interindividuelle due au transport des xénobiotiques.

L'objectif de cet article est de faire la synthèse sur la structure et la fonction de la glycoprotéine-P, produit du gène $A B C B 1$ et impliqué dans le transport des médicaments ; de déterminer les particularités polymorphiques du gène $A B C B 1$ chez les sujets noirs subsahariens et d'énoncer l'impact fonctionnel et les perspectives liées au gène $A B C B 1$ et à la glycoprotéine-P dans le domaine de la pharmacothérapie.

\section{Transport des xenobiotiques}

De par notre environnement, nous sommes amenés à être exposés quotidiennement, directement ou indirectement, à des substances exogènes d'origines diverses, appelées xénobiotiques. Ces composés regroupent des produits naturels, des médicaments et des polluants de l'environnement.

Généralement hydrophobes, ces molécules ont pour tendance naturelle de s'accumuler dans les phases lipidiques des membranes cellulaires. Elles entraîneraient ainsi une mort inéluctable des organismes si ceux-ci ne s'étaient dotés, au cours de l'évolution, de systèmes enzymatiques permettant leur métabolisation et leur élimination. Ces molécules doivent être métabolisées en composés hydrophiles pour être plus facilement éliminés dans la bile et dans les urines (Beaune and Loriot, 2000).

Après administration orale, les médicaments doivent pénétrer dans le compartiment vasculaire pour atteindre leur cible, en franchissant la barrière intestinale, au niveau de laquelle il existe des transporteurs 
destinés à les expulser (représentée par exemple par la glycoprotéine-P, produit $\mathrm{du}$ gène $A B C B 1(M D R 1)$. La glycoprotéine-P, enzyme de phase III, joue un rôle primodial dans l'élimination des conjugués hydrophiles. Elle permet le transport actif des xénobiotiques et des conjugués de la phase II hors de la cellule (Silverman, 1999).

\section{Origine génétique et la notion de Polymorphisme génétique Bases moléculaires}

Comme pour toutes les protéines de l'organisme, la qualité et la quantité des enzymes qui catalysent les réactions de biotransformation des médicaments dépendent majoritairement de l'information portée par le gène qui les code. Or, ces gènes peuvent présenter des anomalies de séquences (ou mutations), telles que des mutations ponctuelles ou SNP (single nucleotide polymorphism), des insertions, des délétions partielles ou totales, des remaniements ou encore des duplications ou amplifications de gènes (Beaune and Loriot, 2000).

Les SNPs sont nombreux dans le génome humain; on estime leur fréquence à environ une altération toutes les 1000 paires de bases, ce qui en fait environ 3.000.000 dans le génome entier. Ils n'ont pas toutes de conséquences fonctionnelles, mais plusieurs milliers ou dizaines de milliers peuvent avoir de telles conséquences (Beaune and Loriot, 2000).

Les différents types d'altération moléculaire induits par les SNPs sont :

- formation d'un codon stop dans une séquence codante ayant pour conséquence la non formation de la protéine ou formation d'une protéine tronquée et inactive ;

- mutation au niveau d'une jonction exonintron nécessaire à l'épissage ayant pour conséquence la formation d'une protéine de structure altérée ;

- mutation au niveau de la séquence protéique dont la conséquence serait variable selon la position de l'altération au niveau de la séquence de la protéine (centre actif, domaine de régulation, etc.) ;
- mutation au niveau du promoteur ou d'une séquence régulatrice dont la conséquence serait l'absence, la réduction ou la stimulation de la transcription du gène ;

- mutation survenant dans une zone non codante (intron) ;

- mutation sans conséquence pour la séquence protéique (en raison de la dégénérescence du code génétique).

Les duplications ou amplifications de gènes conduisent généralement à une surexpression de la protéine alors que les délétions partielles ou totales conduisent plus souvent à l'absence de l'enzyme en modifiant le cadre de lecture (frame-shift) si elles consistent en un non-multiple de 3 bases ou en les respectant (in frame) dans le cas contraire (Beaune, 2007).

$\mathrm{Au}$ total, les mutations qui affectent les gènes des enzymes peuvent être responsables de variations d'expression et/ou d'activité de ces protéines, en entraînant une diminution, voire un déficit d'activité de l'enzyme, une augmentation de l'activité ou encore une absence totale de la protéine enzymatique (Ingelman-Sundberg et al., 1999).

\section{Définition du polymorphisme génétique}

Ces différentes versions d'un même gène définissent des allèles et chaque individu possède deux versions alléliques d'un même gène, identiques ou différentes (l'une héritée de la mère, l'autre héritée du père), déterminant un génotype particulier. L'existence dans la population générale de ces différentes versions alléliques d'un même gène et, par conséquent, de différents génotypes, définit un polymorphisme génétique. Dans la définition classique du polymorphisme génétique s'ajoute une notion de fréquence arbitraire, précisant que l'allèle le moins fréquent présente une fréquence au moins égale à 1\% (Meyer, 1991). Les polymorphismes génétiques des enzymes du métabolisme des médicaments s'expriment dans la population générale sous la forme de différents phénotypes métaboliques, définissant, dans le cas le plus général, deux groupes d'individus dits métaboliseurs limités ou lents (déficit d'activité enzymatique) et 
métaboliseurs extensifs ou rapides (activité enzymatique normale). L'existence de métaboliseurs dits ultrarapides (activité enzymatique augmentée) ou intermédiaires (activité enzymatique réduite) est également reconnue pour certaines enzymes polymorphes (Ingelman-Sundberg et al., 1999). Des études de familles ont permis d'établir que le phénotype limité se transmet généralement sous le mode autosomique récessif, c'est-à-dire que les individus métaboliseurs limités sont, au niveau de leur génotype, homozygotes ou hétérozygotes composites pour un ou deux allèle(s) nonfonctionnel(s) du gène. La fréquence de ces différents phénotypes est variable dans la population en fonction de l'enzyme polymorphe et, pour une même enzyme, variable en fonction de l'origine ethnique ou géographique des populations étudiées (Gaedigk, 2000).

Ces dernières décennies, de nombreux polymorphismes génétiques d'enzymes du métabolisme des médicaments ont été identifiés. Leur polymorphisme d'expression ou de fonction sont mieux connus et documentés. Cependant, la fréquence et le nombre des mutations décrites varient beaucoup d'une enzyme à l'autre. Cette revue se focalise essentiellement sur le gène $A B C B 1$ et la relevance foctionnelle de ses polymorphismes génétiques.

\section{La glycoprotéine- $P$ ( $P$-gp) et le gène $A B C B 1$ \\ Structure}

La glycoprotéine-P (P-gp) est une protéine transmembranaire de poids moléculaire $170 \mathrm{kd}$, membre de la superfamille des transporteurs ABC (ATPbinding cassette) et est encodée par le gène $A B C B 1$, également appelé $M D R 1$ (multidrug resistance). Elle utilise à la manière de la pompe $\mathrm{Na}+\mathrm{K}+$-ATPase, l'énergie apportée par l'hydrolyse de l'ATP pour expulser des peptides endogènes et exogènes hors de la cellule. La P-gp humaine est une protéine phosphorylée et glycosylée contenant 1280 acides aminés faite de deux séquences homologues et symétriques, chaque séquence contenant 6 domaines transmembranaires et un motif ATP-binding (Higgins and Gottesman, 1992). Une représentation de cette structure est donnée par la Figure 1.

\section{Polymorphismes du gène ABCB1 (MDR1)}

Le gène $A B C B 1$ est composé de 28 exons. Son polymorphisme génétique a été identifié pour la première fois au cours d'études in vitro utilisant les cellules tumorales (Kioka et al., 1989). Plusieurs autres groupes, dont Hoffmeyer et al., vont par la suite séquencer toute la région codante du gène MDRI (Hoffmeyer et al., 2000; Cascorbi et al., 2001; Tang et al., 2002). Les travaux de Allabi et al. (2005) ajoutés aux précédents ont permis l'identification d'une quarantaine de SNPs. Les fréquences de ces allèles varient d'une population à une autre (Marzolini et al., 2004).

Le polymorphisme C3435T, quoique synonyme, a été le premier polymorphisme associé à une diminution de l'expression protéique de la P-gp et, par voie de conséquence, de son activité (Hoffmeyer et al., 2000).

Les résultats relatifs à l'impact fonctionnel du C3435T sont contradictoires dans la littérature. D'autres études par la suite (Kim et al., 2001) vont démontrer l'existence d'une forte association entre les deux polymorphismes C3435T et G2677T suggérant alors que la différence fonctionnelle observée peut être attribuée également au deuxième polymorphisme i.e le G2677T (exon 21). Plusieurs autres associations entre différents SNPs du gène $A B C B 1$ seront identifiés par la suite. D'où l'émergence du concept d'haplotype qui déterminerait mieux l'impact fonctionnel. L'existence de différents haplotypes suivant les populations sera ensuite confirmée par différents travaux (Tang et al., 2002; Kroetz et al., 2003 ; Sai et al., 2004).

\section{Particularites polymorphiques du gene $a b c b 1$ chez les sujets noirs subsahariens} L'étude des variants polymorphiques $\mathrm{du}$ gène $A B C B 1 \quad(M D R I)$ et leur 
structuration en haplotypes a été étudiée pour la première fois au sein d'une population noire africaine (Allabi et al., 2005). Trois nouveaux SNPs ont été identifiés : exon 10-57del A (intron 9); exon 10-8T >A (intron 9); 1662G $>\mathrm{C}$ (exon 14) au cours de ces travaux. Les quatre SNPs suivants: IVS6+139C $>$ T, IVS9$44 \mathrm{~A}>\mathrm{G}, 1236 \mathrm{C}>\mathrm{T}$ et $3435 \mathrm{C}>\mathrm{T}$ ont montré un déséquilibre de liaison confirmant ainsi le concept de bloc. 53 haplotypes ont été identifiés en utilisant un algorithme basé sur le maximum de vraisemblance. Le Tableau 1 donne quelques exemples de différences de fréquences alléliques entre une population noire africaine notamment béninoise et autres populations.

Sur la base de ces résultats et autres paramètres de génétique de population, les auteurs ont estimé que la population béninoise paraît homogène et montre un profil haplotypique assez différent de ce qui a été observé jusqu'à présent (Allabi et al., 2005). Cette extrapolation peut être faite à l'ensemble des populations noires africaines quand bien même il existerait des différences pour un certain nombre de SNPs ou haplotypes.

\section{Impact de la glycoproteine-p en pharmacotherapie et perspectives}

La P-gp a été d'abord décrite dans les cellules tumorales où elle contribue au phénomène de résistance multidrogue vis-àvis des agents anticancéreux. Ce phénomène nommé multi-drug resistance (MDR), est défini comme la capacité des cellules exposées à un médicament de développer une résistance manifestée par un efflux accru des médicaments sous l'action d'une protéine membranaire. En effet, il a été observé, dans les cellules présentant une résistance multidrogue, une expression augmentée d'une glycoprotéine de surface qui était accompagnée d'une réduction dans l'accumulation intracellulaire de l'agent cytotoxique (Kartner et al., 1983). D'abord décrite dans les cellules tumorales, cette protéine est largement exprimée dans les tissus sains au niveau de différents organes excréteurs. De ce fait, cette protéine est exprimée majoritairement au niveau des cellules épithéliales des intestins, de la surface des hépatocytes orientés vers le canal biliaire, de la surface apicale de cellules du foie et du pancréas, de la bordure en brosse des tubules proximaux du rein, des cellules endothéliales des capillaires du cerveau et des testicules, des glandes surrénales et du placenta (Fromm, 2002). La colocalisation de la P-gp avec les cytochromes P450, en particulier le CYP3A4 au niveau de l'intestin et du foie suggère que ce transporteur joue un rôle dans la biodisponibilité orale des médicaments et/ou leur répartition dans les différents compartiments cellulaires. Les modèles animaux ont clairement démontré l'importance de la P-gp. Ainsi les souris CF-1 naturellement déficientes en MDR1a ont présenté nettement plus d'effets neurotoxiques suite à l'administration de l'ivermectine, un antiparasitaire substrat de la P-gp. Ces souris déficientes en P-gp accumulaient 80 fois plus d'ivermectine dans le cerveau par rapport aux autres souris et présentaient des signes marqués de neurotoxicité ( Schinkel et al., 1994; Lankas et al., 1997). L'ivermectine est un médicament de choix utilisé dans le traitement de l'onchocercose. Cependant, plusieurs cas d'effets indésirables graves, notamment des encéphalopathies, ont été rapportés dans la littérature (Chippaux et al., 1996 ; Gardon et al., 1997; Boussinesq et al., 1998 ; Boussinesq and Chippaux, 2001). Mais la particularité de l'histoire de l'ivermectine est liée au fait que ces effets indésirables graves survenaient seulement chez certains sujets africains, représentant une faible proportion des patients traités dans cette localité. A la date de 2001, plus de 46 cas de syndrome d'intoxication du système nerveux central (SNC) après administration d'ivermectine, dont 22 décès, avaient été rapportés chez des Camerounais. Boussinesq pensait que tous les cas n'étaient pas rapportés et estimait à 300 , le nombre probable de cas de ce 
syndrome survenu au sein de cette communauté. Or des travaux réalisés avec des souris knock-out ont clairement mis en évidence le rôle clé joué par la P-gp dans la protection du cerveau à l'égard des substances hydrophobes. Certaines races de chiens présentaient, également, un coma encéphalopathique suite à l'administration de l'ivermectine (Hopper et al., 2002). Mais il a été vite démontré que la sensibilité de ces chiens était liée à certaines mutations du gène MDRla (Mealey et al., 2001; Mealey et al., 2002). L'ensemble de ces observations nous amène à émettre l'hypothèse que les effets indésirables graves rapportés dans la littérature chez des sujets camerounais après administration de l'ivermectine pourrait éventuellement être expliquée par la présence d'un profil particulier du gène MDRl $(A B C B 1)$ chez ces sujets.

De quel profil est-il alors question? Le profil block-2 précédemment décrit par Allabi et al.(2005) et exhibant une concentration plus élevée de la phénytoïne prédisposerait-il à une forte sensibilité à l'ivermectine? Rien n'est évident vu la spécificité de substrat parfois relative observée pour certaines enzymes du métabolisme ou du transport des xénobiotiques. Pour l'instant, le génotypage $A B C B 1$ chez les sujets traités à l'ivermectine peut s'avérer utile pour vérifier l'inférence des résultats obtenus chez les souris, les chiens et chez l'homme.

Les expériences avec les souris knockout ont démontré que la $\mathrm{P}$-gp limite l'entrée de certains médicaments comme la ciclosporine, la digoxine et la vinblastine dans le système nerveux central (Schinkel et al., 1996; Schinkel, 1997). Il a aussi été démontré dans un modèle in vitro que la $\mathrm{P}$-gp diminue l'absorption orale du paclitaxel en favorisant son élimination des enterocytes vers la lumière intestinale (Sparreboom et al., 1997). De nombreux substrats médicamenteux de la P-gp ont été caractérisés, tels que des immunosuppresseurs, des hormones stéroïdiennes, des inhibiteurs calciques, ou encore des antiprotéases.

Les études réalisées par Fromm (2002), Kim (2002) et Marzolini et al., (2004) ont permis de dresser une liste exhaustive des substrats, inhibiteurs ou inducteurs de la gycoprotéine-P dans le Tableau 2.

Cependant chez l'homme, le rôle de la P-gp intestinale dans l'absorption des médicaments est souvent déduite indirectement des études d'inhibition. L'implication de la P-gp dans le processus d'absorption est difficile à prouver directement (Lin and Yamazaki, 2003).

Par exemple, la coadministration du paclitaxel avec la ciclosporine, cette dernière étant à la fois substrat et inhibiteur de la P-gp, augmente significativement la biodisponibilité orale du paclitaxel (Meerum Terwogt et al., 1999). La P-gp serait également impliquée dans le processus d'excrétion de certains médicaments (Mayer et al., 1996 ; Sai et al., 2003). Les processus d'inhibition ou d'induction de la P-gp, quoique nettement moins étudiés par rapport à ceux des CYPs ont montré leur importance clinique en terme d'efficacité et de toxicité du traitement. Plusieurs interactions ont été décrites: par exemple, entre la digoxine et d'autres médicaments comme le vérapamil, la quinidine et l'amiodarone (Pedersen 1985; Verschraagen et al., 1999). Il a aussi été montré que l'administration concomitante de vérapamil et de digoxine augmentait la concentration plasmatique de cette dernière de $40 \%$ résultant probablement d'une inhibition intestinale, hépatique ou rénale de la P-gp. Au total, la P-gp, en raison de ses localisations stratégiques, a pour fonction de limiter l'absorption des xénobiotiques par le tractus gastro-intestinal, de promouvoir leur efflux dans l'urine et dans la bile et de jouer un rôle protecteur pour le cerveau et le fœetus.

L'émergence du concept d'haplotype permet de mieux déterminer l'impact fonctionnel du gène $A B C B 1$. Toutefois, avant l'émergence du concept d'haplotype, plusieurs études ont montré déjà que le gène MDRl pouvait être un facteur 
prédictif de la réponse aux médicaments. Par exemple, chez les malades infectés par le VIH et traités par des inhibiteurs de protéase (nelfinavir, efavirenz), le génotype 3435T/T était associé à une meilleure restauration immune (évaluée par le taux de cellules CD4+) et des concentrations plasmatiques en anti protéases moins élevées (Fellay et al., 2002). De récents travaux ont confirmé les rôles des polymorphismes $T-129 C$ et G2677A du gène $A B C B 1$ dans la restauration immune des patients infectés par le VIH et sous traitement antirétroviral dans une population sud-africaine (Parathyras et al., 2009).

De la même manière, l'impact du génotype $A B C B 1$ a été également étudié dans le cadre de traitements immunosuppresseurs par la ciclosporine ou le tacrolimus, qui sont substrats de la P-gp, notamment chez les transplantés rénaux (Saeki et al., 1993; Schinkel et al., 1996; Herrero et al., 2010 ; Shi et al., 2011). Le polymorphisme de ABCB1 a été corrélé à une modification de la pharmacocinétique et, en particulier, de la clairance orale de la ciclosporine, qui est augmentée chez les sujets porteurs de la mutation 3435T. Plusieurs études récentes ont montré que la posologie nécessaire pour l'obtention d'une concentration en zone thérapeutique, un mois après la transplantation, est bien corrélée au polymorphisme $\mathrm{ABCB} 1$ et que le génotype $A B C B 1$ constitue un facteur prédictif de la dose initiale optimale de tacrolimus, avec une réduction de $40 \%$ de la posologie nécessaire chez les patients homozygotes mutés (Macphee et al., 2002; Anglicheau et al., 2003). Cette association entre le génotype $A B C B 1$ et la dose nécessaire de tacrolimus est toutefois loin d'être évidente selon d'autres auteurs (Haufroid et al., 2004). Néammoins, le profil haplotypique particulier exhibé par les sujets de race noire peut expliquer la différence de profil pharmacocinétique pour la ciclosporine observée entre les sujets de race noire et les caucasiens.

Dans le cadre d'un traitement au long cours avec le clopidogrel, il a été trouvé une association significative entre le polymorphisme $(3435 \mathrm{C} / \mathrm{T})$ et le décès par maladie cardiovasculaire, la survenue de l'infactus du myocarde ou des accidents vasculocérébraux (Mega et al., 2010).

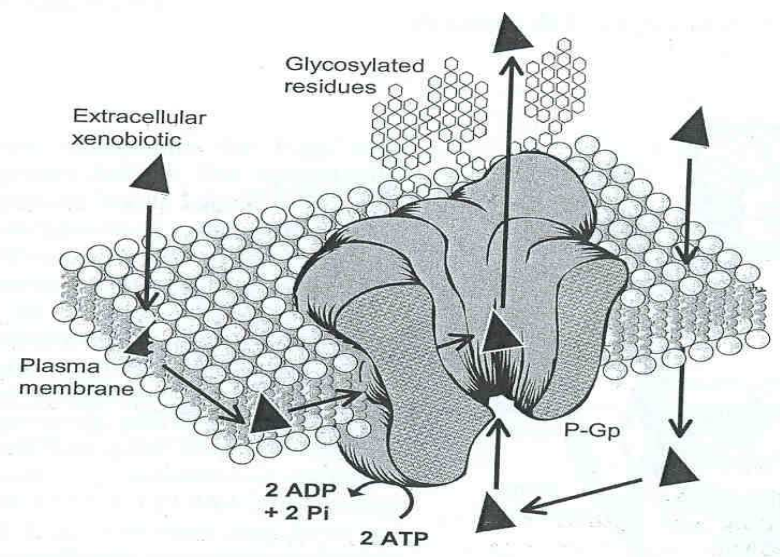

Figure 1: Structure de la glycoprotéine-P.

(Figure obtenue après autorisation de Marzolini et al, 2004). 
A. C. ALLABI et al. / Int. J. Biol. Chem. Sci. 6(2): 844-863, 2012

Tableau 1: Polymorphisme génétique en $A B C B 1$ : population béninoise versus d'autres ethnies.

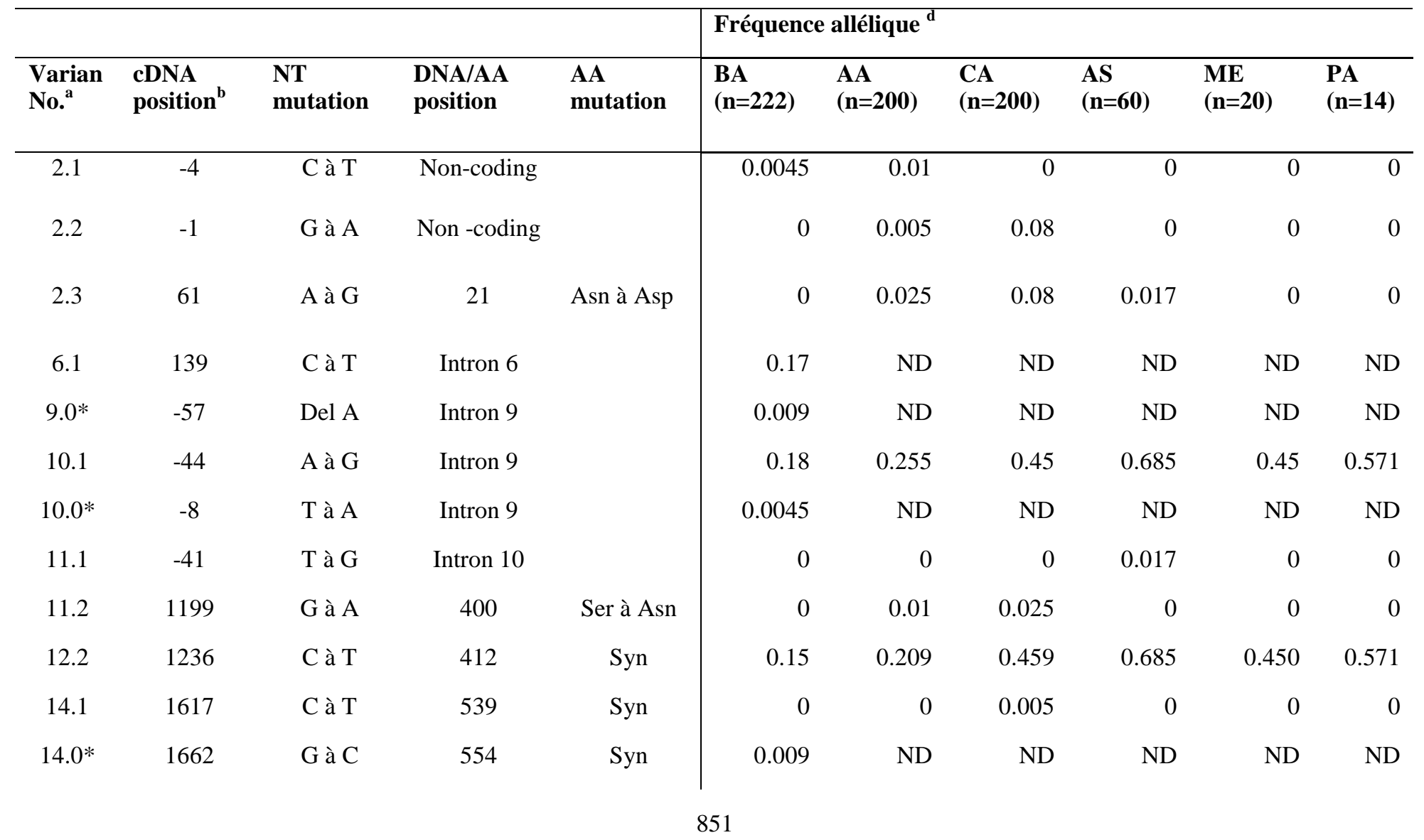


A. C. ALLABI et al. / Int. J. Biol. Chem. Sci. 6(2): 844-863, 2012

\begin{tabular}{ccccc|rrrrrr}
14.2 & 38 & A à G & Intron 14 & & 0.49 & 0.54 & 0.505 & 0.683 & 0.45 \\
17.1 & -76 & T à A & Intron 16 & & 0.347 & ND & ND & ND & ND & ND \\
21.2 & 2650 & C à T & 884 & Syn & 0 & 0.005 & 0.005 & 0 & 0 \\
$21.3 \mathrm{a}$ & 2677 & G à T & 893 & Ala à Ser & 0.009 & 0.1 & 0.464 & 0.45 & 0.4 & 0.357 \\
$21.3 \mathrm{~b}$ & 2677 & G à A & 893 & Ala à Thr & 0 & 0.005 & 0.036 & 0.067 & 0 & 0.357 \\
26.2 & 3421 & T à A & 1141 & Ser à Thr & 0.054 & 0.111 & 0 & 0 & 0.05 \\
26.3 & 3435 & C à T & 1145 & Syn & 0.14 & 0.202 & 0.561 & 0.4 & 0.5 & 0.5 \\
\hline
\end{tabular}

${ }^{a}$ Les Variants sont comptés sequentiellement par exon. Par exemple, le variant 2.1 est le premier variant dans l'exon 2. Les nouveaux variants sont indiqués par (*).

${ }^{b}$ La position cDNA est déterminée en relation avec le début du site de translation ATG et basée sur la séquence cDNA du GenBank ayant pour référence M14758 avec un A comme référence nt à la position -43 .

'La localisation des variants dans la séquence du DNA est indiquée comme intronique ou non-codante; intron -1 est la séquence intronique précédant immediatement l'exon 1. La position des acides aminés (AA) est indiquée pour les variants se situant dans la séquence exonique codante.

¿Les fréquences alléliques sont calculées pour la population totale et pour chaque groupe ethnique; BA Noirs Africains d'origine beninoise, AA, Afro-Americains, CA,

Causasiens-Americains, AS, Americains d'origine Asiatique, ME, Americains d'origine mexicaine, PA, Originaire des îles du Pacifique. $n$ est le le nombre de chromosomes dans chaque groupe ethnique; ND, non-détectée.

Les données des autres ethnies proviennent de Kroetz et al., 2003. 
Tableau 2 : Liste de quelques médicaments, substrats, inducteurs ou inhibiteurs de la glycoprotéine-P.

\begin{tabular}{lccc}
\hline \multicolumn{1}{c}{ Médicament } & Substrat & Inhibiteur & Inducteur \\
\hline Immunosuppresseurs & $\sqrt{ }$ & $\sqrt{ }$ & \\
Cyclosporine & $\sqrt{ }$ & $\sqrt{ }$ & \\
Sirolimus & $\sqrt{ }$ & $\sqrt{ }$ & \\
Tacrolimus & $\sqrt{ }$ & & \\
Valspodar & & & \\
Antidépresseurs & $\sqrt{ }$ & $\sqrt{ }$ & \\
Amitriptyline & & $\sqrt{ }$ & \\
Fluoxetine & & & \\
Paroxetine & & & \\
Sertraline & & $\sqrt{ }$ & \\
St John's wort & & $\sqrt{ }$ \\
Neuroleptiques & & \\
Chlorpromazine & & \\
Flupenthixol & & \\
Phenothiazine & & \\
& & \\
Antiépileptiques & & \\
Phenibarbital & & \\
Phennytoin & $\sqrt{ }$ & \\
Antiacides & & \\
Cimetidine & & \\
Ranitidine & &
\end{tabular}

\section{Oploïdes}

Methadone

Morphine

Pentazocine

Antiémétiques

Domperidon

Ondansetron

$\sqrt{ }$
$\sqrt{ }$

\section{Antidiarrhéiques}

Loperamide

\section{Hypolipidéiques}

Artovastatine

Lovastatine

\section{Antihistaminiques H1}

Fexofenadine 
Terfénadine

$\sqrt{ }$

Antiparasitaires

Ivermectine

Méfloquine

$\sqrt{ }$

Autres

Bromocriptine

Colchicine

Dipyridamole

Emetine

Progesterone

Acide rétinoïque

rhodamine

spironolactone

$\sqrt{ }$

$\overline{\text { (Données obtenues en compilant les revues de la littérature de Marzolini et al, 2004; Fromm, } 2002 \text { et Kim, 2002) }}$

Tableau 2 (suite) : Liste de quelques médicaments, substrats, inducteurs ou inhibiteurs de la glycoprotéine-P.

\begin{tabular}{|c|c|c|c|}
\hline Médicament & Substrat & Inhibiteur & Inducteur \\
\hline \multicolumn{4}{|l|}{ Anticancéreux } \\
\hline Actinomycin & $\sqrt{ }$ & & \\
\hline Daunorubicin & $\sqrt{ }$ & & \\
\hline Docetaxel & $\sqrt{ }$ & & \\
\hline Etopoxide & $\sqrt{ }$ & & \\
\hline Imatinib & $\sqrt{ }$ & & \\
\hline Irinotecan & $\sqrt{ }$ & & \\
\hline Mitomycin C & $\sqrt{ }$ & & \\
\hline Paclitaxel & $\sqrt{ }$ & & \\
\hline Teniposide & $\sqrt{ }$ & & \\
\hline Topotecan & $\sqrt{ }$ & & \\
\hline Vinblastine & $\sqrt{ }$ & & \\
\hline Vincristine & $\sqrt{ }$ & & \\
\hline
\end{tabular}

\section{Antihypertenseurs}

Carvediol

Celiprolol

Dilitiazem

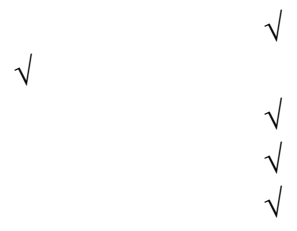

$\sqrt{ }$

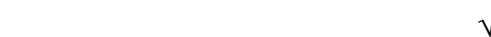

$\sqrt{ }$ 
A. C. ALLABI et al. / Int. J. Biol. Chem. Sci. 6(2): 844-863, 2012

Nicardipine

Reserpine

Talinolol

Bunitrolol

Celiprolol

Mibefradil (inhibiteur)

D-617

D-620

\section{Antiarrhytmiques}

Amiodarone

Digoxine

Propafenone

Quinidine

Verapamil

\section{Glucocorticoides}

Aldosterone

$\sqrt{ }$

Cortisol

Dexamethasone

Metylprednisolone

\section{Inhibiteurs protéasiques HIV}

Amprenavir
Indinavir
Nelfinavir
Saquinavir
Ritonavir

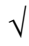

$\sqrt{ }$

$\sqrt{ }$

$\sqrt{ }$

$\sqrt{ }$

\section{tiques}

Clarithromycine

Erythromicine

Levofloxacine

Rifampicine

Sparfloxacine

$\begin{array}{ll} & \sqrt{ } \\ \sqrt{ } & \sqrt{ } \\ \sqrt{ } & \\ \sqrt{ } & \end{array}$




\section{Antimycotiques}

Itraconazole

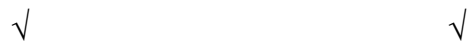

ketoconazole

(Données obtenues en compilant les revues de la littérature de Marzolini et al, 2004; Fromm, 2002 et Kim, 2002).

Par ailleurs, les polymorphismes du gène $A B C B 1$ ont été associés à une modification de la pharmacocinétique de la quinine et ainsi à la susceptibilité aux effets indésirables liés à la quinine (Mukonzo et al., 2010).

Enfin, la résistance aux glucocorticoïdes utilisés dans la maladie de Crohn semble dépendre du niveau d'expression de la P-gp. Une expression lymphocytaire plus élevée de la P-gp a en effet été retrouvée chez les malades non répondeurs et nécessitant un traitement chirurgical (Farrell et al., 2000).

\section{Modulation de l'expression de la P-gp en Pharmacothérapie}

Des données ont démontré la possibilité de moduler le niveau d'expression de la glycoprotéine-P. Ainsi le groupe de Kim et al. a montré une meilleure pénétration intracérébrale et une plus forte biodisponibilité des inhibiteurs de protéases suite à la coadministration de LY335979, un inhibiteur de P-g. Les inhibiteurs de protéases (indinavir, saquinavir, nelfinavir) connus pour être des substrats de la glycoprotéine-P (Kim et al., 1998; Washington et al., 1998; Profit et al., 1999) peuvent être ainsi boostés ou coadministrés avec des inhibiteurs de P-gp afin d'augmenter leur biodisponibilité ou leur concentration intracérébrale. Ces stratégies seront bien entendues envisagées selon le statut génotypique $A B C B I$ des patients.

Cette option d'utilisation des modulateurs de la $\mathrm{P}$-gp peut être également envisagée pour le suivi thérapeutique des patients sous antidépresseurs. En effet, il a été trouvé une association significative entre l'expression de la glycoprotéine-P et la concentration intracérébrale des antidépresseurs (O'Brien et al., 2011). Il est alors envisageable en cas de résistance au traitement d'augmenter la biodisponibilité ou la concentration des molécules au site d'action en utilisant ces modulateurs de l'expression de la P-gp. Dans ces circonstances, on peut également changer d'antidépresseur en choisissant une molécule ayant davantage d'action inhibitrice sur l'expression de la P-gp.

Mais toutes ces stratégies thérapeutiques méritent d'être évaluées aux cours de différents études cliniques.

Un nombre important de chercheurs s'intéressent au rôle joué par les polymorphismes du gène $A B C B 1$ dans la survenue de l'hépatotoxicité associée à certains antiretroviraux (Kim, 2003 ; Sanchez Hellin and Gutiérrez Rodero, 2008 ; Ciccacci et al., 2010) vu leur contribution à la restauration immunitaire chez les patients infectés par le VIH. Globalement, Il urge, dans le cadre de différentes études, à venir, d'évaluer l'importance du gène $A B C B 1$ par rapport à d'autres comme le $C Y P 2 B 6, C Y P 3 A 4 / 5$, SLCO1B3 etc.. (Kedmi et al., 2007 ; Lakhman et al., 2009 ; Chantarangsu et al., 2009 ; Rakhmanina et al., 2011 ; Chen et al., 2010 ; Kwara et al., 2011) dans la pharmacogénétique du VIH. L'intégration des paramètres pharmacogé-nétiques comme covariables dans des modèles de 
pharmacocinétique-pharmacodynamie sont incontestablement des outils de demain pour mieux comprendre les interactions médicamenteuses et optimiser les bénéfices thérapeutiques.

L'intérêt des polymorphismes du gène $A B C B 1$ ansi donc de l'expression de la $\mathrm{P}$-gp en cancérologie n'est plus à démontrer ( Deenen et al., 2011) et continue d'être une obsession pour de nombreux chercheurs pour optimiser le suivi thérapeutique en diminuant par exemple les effets indésirables. L'intérêt du gène $A B C B 1$ pour plusieurs classes thérapeutiques telles les analgésiques (Nawa et al., 2011), les antiagrégants plaquetaires (Mega et al., 2010), les antihypertenseurs (Eap et al., 2007 ; Valente et al., 2007 ; Bochud et al., 2009 ; Yanagimachi et al., 2010), les antiépileptiques (Allabi et al., 2005) restent également d'actualité.

Les progrès récents de la pharmacogénétique notamment chez les sujets de race noire devraient conduire à une adaptation de la prescription chez ces sujets, tant pour augmenter l'efficacité que pour réduire les risques d'effets indésirables.

Une étude prospective étudiant la relation entre effets indésirables et pharmacogénétique chez les noirs subsahariens devrait mieux élucider les impacts fonctionnels des différents polymorphismes spécifiques à cette race et aider à mieux prévenir les risques d'interactions médicamenteuses. Ces différences inter-ethniques dans la pharmacocinétique et la pharmacodynamie de certains médicaments doivent être prises en considération par les agences administratives d'autorisation de mise sur le marché des médicaments notamment lors de la phase d'interprétation des essais cliniques réalisés dans différentes régions du monde. Les taux de succès ou échecs thérapeutiques ne sont pas nécessairement extrapolables d'une région à une autre. C'est la raison pour laquelle la Conférence Internationale de l'harmonisation (ICH, "The International Conference on Harmonization") a publié un guide dans lequel il recommande l'évaluation de facteurs ethniques sur la pharmacodynamie ou la toxicité de la molécule (Kim et al., 2004).

Toutefois, si le gène $A B C B 1$ explique une partie de la variabilité interindividuelle de la pharmacocinétique ou pharmacodynamie des médicaments, une large proportion de celle-ci reste inexpliquée. L'implication d'autres protéines dans le transport des xénobiotiques ne peut être totalement exclue. De nombreuses protéines interviennent, par exemple, dans le processus d'absorption et peuvent interférer sur la glycoprotéine-P ou sur les CYPs présents au niveau intestinal. Ainsi les membres des familles MRP ou OATP (Organic Anion Transporting Polypeptide) peuvent interférer sur le processus du transport de nombreux médicaments ou xénobiotiques en exerçant un effet inverse à celui de la P-gp. Il n'est pas exclu aussi que d'autres enzymes de phase I ou phase II contribuent à expliquer cette variabilité interindividuelle. La P-gp et les CYP3A4/5 peuvent agir en synergie dans certains tissus afin de prévenir l'entrée des xénobiotiques dans la circulation systémique .

\section{REFERENCES}

Allabi AC, Horsmans Y, Issaoui B, Gala JL. 2005. Single nucleotide polymorphisms of ABCB1 (MDR1) gene and distinct haplotype profile in a West Black African population. Eur J Clin Pharmacol, 61(2): 97-102. 
Anglicheau D, Verstuyft C, Laurent-Puig P, Becquemont L, Schlageter MH, Cassinat $\mathrm{B}$, Beaune $\mathrm{P}$, Legendre $\mathrm{C}$, Thervet $\mathrm{E}$. 2003. Association of the multidrug resistance-1 gene single-nucleotide polymorphisms with the tacrolimus dose requirements in renal transplant recipients. J Am Soc Nephrol, 14(7): 1889-1896.

Beaune P, Loriot MA. 2000. Bases moléculaires de la susceptibilité aux xénobiotiques: aspects métaboliques. Médecine/Sciences, 16(1): 1051-1056.

Beaune PH. 2007. Pharmacogenetics:from basic research to clinical applications. Ann Pharm Fr, 65(6): 365-370.

Beutler E. 1993. Study of glucose-6phosphate dehydrogenase: history and molecular biology. Am J Hematol, 42(1): 53-55.

Bochud M, Bovet P, Burnier M, Eap CB. 2009. CYP3A5 and ABCB1 genes and hypertension. Pharmacogenomics, 10(3): 477-487.

Bodin K, Bretillon L, Aden Y, Bertilsson L, Broome U, Einarsson C, Diczfalusy U. 2001.Antiepileptic drugs increase plasma levels of 4beta-hydroxycholesterol in humans: evidence for involvement of cytochrome p450 3A4. J Biol Chem, 276(42): 38685-38689.

Boussinesq M, Chippaux JP. 2001. A controlled prospective trial of the prophylactic effect of a single dose of ivermectin against Onchocerca volvulus. Parasite, 8(3):255-259.

Boussinesq M, Gardon J, Gardon-Wendel N, Kamgno J, Ngoumou P, Chippaux JP. 1998. Three prob able cases of Loa loa encephalopathy following ivermectin treatment for onchocerciasis. Am J Trop Med Hyg, 58(4): 461-469.
Burgoine KL, Bancone G, Nosten F. 2010. The reality of using primaquine. Malar $J$, 9: 376 .

Cascorbi I, Gerloff T, Johne A, Meisel C, Hoffmeyer S, Schwab M, Schaeffeler E, Eichelbaum M, Brinkmann U, Roots I. 2001. Frequency of single nucleotide polymorphisms in the P-glycoprotein drug transporter MDR1 gene in white subjects. Clin Pharmacol Ther, 69(3): 169-174.

Chantarangsu S, Cressey TR, Mahasirimongkol S, Capparelli E, Tawon Y, Ngo-Giang-Huong N, Jourdain G, Lallemant M, Chantratita W. 2009. Influence of CYP2B6 polymorphisms on the persistence of plasma nevirapine concentrations following a single intrapartum dose for the prevention of mother to child transmission in HIV-infected Thai women. J Antimicrob Chemother, 64(6): 1265-1273.

Chen J, Sun J, Ma Q, Yao Y, Wang Z, Zhang L, Li L, Sun F, Lu H. 2010. CYP2B6 polymorphism and nonnucleoside reverse transcriptase inhibitor plasma concentrations in Chinese HIV-infected patients. Ther Drug Monit, 32(5): 573578.

Chippaux JP, Boussinesq M, Gardon J, Gardon-Wendel N, Ernould JC. 1996. Severe adverse reaction risks during mass treatment with ivermectin in loiasisendemic areas. Parasitol Today, 12(11): 448-450.

Ciccacci C, Borgiani P ,Ceffa S, Sirianni E, Marazzi MC, Altan AM, paturzo G, Bramanti P, ovelli G, Pablombi L. 2010. Niverapin- induced hepatoxicity and pharmacogenetics : A retrospective study in a population from Mozambique. Pharmacogenomics, 11(1): 23-31. 
Daly AK, King BP. 2003. Pharmacogenetics of oral anticoagulants. Pharmacogenetics, 13(5): 247-252.

Deenen MJ, Cats A, Beijnen JH, Schellens JH . 2011. Part2:pharmacogenetic variability in drug transport and phase I anticancer drug metabolism. Oncologist, 16(6): 820-834.

Eap CB, Bochud M, Elston RC, Bovet P, Maillard MP, N ussberger J, Schild L, Shamlaye C, Burnier M . 2007. CYP3A5 and $\mathrm{ABCB} 1$ genes influence blood pressure and response to treatment, and their effect is modified by salt. Hypertension, 49(5): 1007-1014.

Farrell RJ, Murphy A, Long A, Donnelly S, Cherikuri A, O'Toole D, Mahmud N, Keeling PW, Weir DG, Kelleher D. 2000. High multidrug resistance (Pglycoprotein 170) expression in inflammatory bowel disease patients who fail medical therapy. Gastroenterology, 118(2): 279-288.

Fekih L, Boussoffara L, Fenniche S, Abdelghaffar H, Megdiche ML. 2011. Neuropsychiatric side effects of antituberculosis agents. Rev Med Liege, 66(2): 82-85.

Fellay J, Marzolini C, Meaden ER, Back DJ, Buclin T, Chave JP, Decosterd LA, Furrer H, Opravil M, Pantaleo G, Retelska D, Ruiz L, Schinkel AH, Vernazza P, Eap CB, Telenti A. 2002. Response to antiretroviral treatment in HIV-1-infected individuals with allelic variants of the multidrug resistance transporter 1: a pharmacogenetics study. Lancet, 359(9300): 30-36.

Fromm MF. 2002. The influence of MDR1 polymorphisms on P-glycoprotein expression and function in humans. $A d v$ Drug Deliv Rev, 54(10): 1295-1310.
Gaedigk A. 2000. Interethnic differences of drug-metabolizing enzymes. Int $J$ Clin Pharmacol Ther, 38(2): 61-68.

Gardon J, Gardon-Wendel N, Demanga N, Kamgno J, Chippaux JP, Boussinesq M. 1997. Serious reactions after mass treatment of onchocerciasis with ivermectin I $\mathrm{n}$ an area endemic for Loa loa infection. Lancet, 350(9070): 18-22.

Haufroid V, Mourad M, Vankerckhove V, Wawrzyniak J, De Meyer M, Eddour DC, Malaise J, Lison D, Squifflet JP, Wallemacq P. 2004. The effect of CYP3A5 and MDR1 polymorphisms on cyclosporine and tacrolimus dose requirements and through blood levels in stable renal transplant patients. Pharmacogenetics, 14(3): 147-154.

Herrero MJ, Sánchez-Plumed J, Galiana M, Bea S, Marqués MR, Aliño SF. 2010. Influence of pharmacogenetic polymorphisms in routine immunosuppression therapy after renal transplantation. Transplant Proc, 42(8): 3134-3136.

Higgins CF, Gottesman MM. 1992. Is the multidrug transporter a flippase? Trends Biochem Sci, 17(1): 18-21.

Hoffmeyer S, Burk O, von Richter O, Arnold HP, Brockmoller J, Johne A, Cascorbi I, Gerloff T, Roots I, Eichelbaum M, Brinkmann U. 2000. Functional polymorphisms of the human multidrugresistance gene: multiple sequence variations and correlation of one allele with P-glycoprotein expression and activity in vivo. Proc Natl Acad Sci $U S$ A, 97(7): 3473-3478.

Hopper K, Aldrich J, Haskins SC. 2002. Ivermectin toxicity in 17 collies. $\mathrm{J}$ Vet Intern Med, 16(1): 89-94.

Ingelman-Sundberg M ,Oscarson M, McLellan RA. 1999. Polymorphic human cytochrome P450 enzymes: opportunity 
for individualized drug treatment. Trends Pharmacol Sci, 20(8): 342-349.

Jamshidi Y, Moreton M, McKeown DA, Andrews S,Nithiyananthan T, Tinworth L, Holt DW, Sadiq ST /. 2010. Tribal ethnicity and CYP2B6 genetics in Ugandan and Zimbabwean populations in the UK : implications for efavirenz dosing in HIV infection. J Antimicrob Chemother, 65(12): 1614-1629.

Jeovanio-Silva AL, Monteiro TP, El-Jaick $\mathrm{KB}$, do Brasil PE, Rolla VC, de Castro L . 2012. Unique CYP3A4 genetic variant in Brazilian tuberculosis patients with/without HIV. Mol Med Rep, 5(1): 153-156.

Kedmi M, Maayan S, Cohen SB, Hauzi M, Rund D . 2007. MDR1 and CYP3A4 polymorphisms are associated with HIV seropositivity in Israeli patients but do not influence the course of HIV disease. AIDS Patient Care STDS, 21(9): 653-658

Kim K, Johnson JA, Derendorf H. 2004. Differences in drug pharmacokinetics between East Asians and Caucasians and the role of genetic polymorphisms. J Clin Pharmacol, 44(10): 1083-1105.

Kim RB . 2003. Drug transporters in HIV therapy. Top HIV Med, 11(4): 136-139.

Kim RB, Fromm MF, Wandel C, Leake B, Wood AJ, Roden DM, Wilkinson GR . 1998. The drug transporter -glycoprotein limits oral absorption and brain entry of HIV-1 protease inhibitors. J Clin Inv Est, 101(2): 289-294.

Kim RB, Leake BF, C hoo EF, Dresser GK, Kubba SV, Schwarz UI, Taylor A, Xie HG, McKinsey J, Zhou S, Lan LB, Schuetz JD, Schuetz EG, Wilkinson GR. 2001. Identification of functionally variant MDR1 alleles among European Americans and African Americans. Clin Pharmacol Ther, 70(2): 189-199.
Kioka N, Tsubota J, Kakehi Y, Komano T, Gottesman MM, Pastan I, Ueda K: Pglycoprotein gene (MDR1) cDNA from human adrenal. 1989. normal Pglycoprotein carries Gly185 with an altered pattern of multidrug resistance. Biochem Biophys Res Commun, 162(1): 224-231.

Kroetz DL, Pauli-Magnus C, Hodges LM, Huang CC, Kawamoto M, Johns SJ, Stryke D, Ferrin TE, DeYoung J, Taylor T, Carlson EJ, Herskowitz I, Giacomini KM, Clark AG. 2003. Sequence diversity and haplotype structure in the human ABCB1 (MDR1, multidrug resistance transporter) gene. Pharmacogenetics, 13(8): 481-494.

Kwara A, Lartey M, Sagoe KW, Court MH : 2011. Paradoxically elevated efavirenz concentrations in HIV/tuberculosiscoinfected patients with CYP2B6 516TT genotype on rifampin-containing antituberculous therapy: $A I D S, \quad \mathbf{2 5}(3)$ : 388-390.

Lakhman SS, Ma Q, Morse GD. 2009. Pharmacogenomics of CYP3A: consideration for hiv treatment. Pharmacogenomics, 10(8): 1323-1339.

Lankas GR, Cartwright ME, Umbenhauer D. 1997. P-glycoprotein deficiency in a subpopulation of CF-1 mice enhances avermectin-induced neurotoxicity. Toxicol Appl Pharmacol, 143(2): 357365.

Lazarou J, Pomeranz BH, Corey PN. 1998. Incidence of adverse drug reactions in hospitalized patients: a meta-analysis of prospective studies. Jama, 279(15): 12001205.

Lin JH, Yamazaki M .2003. Role of Pglycoprotein in pharmacokinetics: clinical implications. Clin Pharmacokinet, 42(1): 59-98. 
Macphee IA, Frederi cks S, Tai T, Syrris P, Carter ND, Johnston A, Goldberg L, Holt DW. 2002. Tacrolimus pharmacogenetics: polymorphisms associated with expression of cytochrome p4503A5 and P-glycoprotein correlate with dose requirement. Transplantation, 74(11): 1486-148.

Marzolini C, Paus E, Buclin T, Kim RB. 2004. Polymorphisms in human MDR1 (P-glycoprotein): recent advances and clinical relevance. Clin Pharmacol Ther, 75(1): 13-33.

Mayer U, Wagenaar E, Beijnen JH, Smit JW, Meijer DK, van Asperen J, Borst P, Schinkel AH. 1996. Substantial excretion of digoxin via the intestinal mucosa and prevention of long-term digoxin accumulation in the brain by the mdr 1a P-glycoprotein. Br J Pharmacol, 119(5): 1038-1044.

Mealey KL, Bentjen SA, Gay JM, Cantor GH. 2001. Ivermectin sensitivity in collies is associated with a deletion mutation of the mdr1 gene. Pharmacogenetics, 11(8): 727-733.

Mealey KL, Bentjen SA, Waiting DK. 2002. Frequency of the mutant MDR1 allele associated with ivermectin sensitivity in a sample population of collies from the northwestern United States. Am J Vet Res, 63(4): 479-481.

Meerum Terwogt JM, Malingre MM, Beijnen $\mathrm{JH}$, ten Bokkel Huinink WW, Rosing H, Koopman FJ, van Tellingen O, Swart M, Schellens JH. 1999. Coadministration of oral cyclosporin A enables oral therapy with paclitaxel. Clin Cancer Res, 5(11): 3379-3384.

Mega JL, Close SL, Wiviott SD, Shen L, Walker JR, Simon T, Antman EM, Braunwald E, Sabatine MS. 2010. Genetic variants in ABCB1 and CYP2C19 and cardiovascular outcomes after treatment with clopidogrel and prasugrel in the TRITON-TIMI 38 trial: a pharmacogenetic analysis. Lancet, 376(9749): 1312-1319.

Mukonzo JK, Waako P, Ogwal-Okeng J, Gustafsson LL, Aklillu E. 2010. Genetic variations in $\mathrm{ABCB} 1$ and $\mathrm{CYP} 3 \mathrm{~A} 5$ as well as sex influence quinine disposition among Ugandans. Ther Drug Monit, 32(3): 346-352.

Nawa A, Fujita-Hamabe W, Kishioka S, Tokuyama S. 2011. Decreased expression of intestinal P-glycoprotein increase the analgesic effects of oral morphine in a streptozotocin-induced diabetic mouse model . Drug Metab Pharmacokinet, Aug 30 .

Ngaimisi E, Mugusi S, Minzi O, Sasi P, Riedel KD, Suda A, Ueda N, Janabi M, Mugusi F, Haefeli WE, Bertilsson L, Burhenne J, Aklillu E. 2011. Effect of rifampicin and CYP2B6 genotype on long-term efavirenz autoinduction and plasma exposure in HIV patients with or without tuberculosis. Clin Pharmacol Ther, 90(3): 406-413 .

O'Brien FE, Dinan TG, Griffin BT, Cryan JF. 2011. Interactions between antidepressants and P-glycoprotein at the bloodbrain barrier: Clinical significance of in vitro and in vivo findings. $\mathrm{Br} J$ Pharmacol, 165(2): 289-312.

Oscarson M. 2003. Pharmacogenetics of drug metabolising enzymes: importance for personalised medicine. Clin Chem Lab Med, 41(4): 573-580.

Pantuck EJ. 1993. Plasma cholinesterase: gene and variations. Anesth Analg, 77: 380386.

Parathyras J, Gebhardt S, Hillermann-Rebello R, Grobbelaar N, Venter M, Warnich L. 2009. Apharmacogenetic study of CD4 recovery in response to HIV antiretroviral 
therapy in two South African population groups. J Hum Genet, 54:261-265.

Pedersen KE. 1985. Digoxin interactions. The influence of quinidine and verapamil on the pharmacokinetics and receptor binding of digitalis glycosides. Acta Med Scand Suppl, 697:1-40.

Pouyanne P, Haramburu F, Imbs JL, Begaud B. 2000. Admissions to hospital caused by adverse drug reactions: cross sectional incidence study. French Pharmacovigilance Centres. Bmj, 320(7241): 1036.

Profit L, Eagling VA, Back DJ. 1999. Modulation of P-glycoprotein function in human lymphocytes and Caco-2 cell monolayers by HIV-1 protease inhibitors. Aids, 13(13): 1623-1627.

Rakhmanina NY, Neely MN, Van Schaik RH, Gordish-Dressman HA, Williams KD, Soldin SJ, van den Anker JN. 2011.CYP3A5, ABCB1, and SLCO1B1 polymorphisms and pharmacokinetics and virologic outcome of lopinavir/ritonavir in HIV-infected children. Ther Drug Monit, 33(4): 417424.

Saeki T, Ueda K, Tanigawara Y, Hori R, Komano T. 1993. Human P-glycoprotein transports cyclosporin A and FK506. $J$ Biol Chem, 268(9): 6077-6080.

Sai K, Kaniwa N, Itoda M, Saito Y, Hasegawa R, Komamura K, Ueno K, Kamakura S, Kitakaze M, Shirao K, Minami H, Ohtsu A, Yoshida T, Saijo N, Kitamura Y, Kamatani N, Ozawa S, Sawada J. 2003. Haplotype analysis of ABCB1/MDR1 blocks in a Japanese population reveals genotype-dependent renal clearance of irinotecan. Pharmacogenetics, 13(12): 741-757.

Sanchez Hellin V, Gutiérrez Rodero F. 2008. Toxicogenetics of antirétroviral treatment (II) : neurotoxicity, hepatoxicity, lactic acidosis, kidney damage, and others advers effets of antiretrovira drugs. Enferm Infect Microbiol Clin, 6: 24-33.

Schinkel AH, Wagenaar E, Mol CA, van Deemter L. 1996. P-glycoprotein in the blood-brain barrier of mice influences the brain penetration and pharmacological activity of many drugs. J Clin Invest, 97(11): 2 517-2524.

Schinkel AH. 1997. The physiological function of drug-transporting Pglycoproteins. Semin Cancer Biol, 8(3): 161-170.

Shi XJ, Geng F, Jiao Z, Cui XY, Qiu XY, Zhong MK. 2011. Association of ABCB1, CYP3A4*18B and CYP3A5*3 genotypes with the pharmacokinetics of tacrolimus in healthy Chinese subjects: A population pharmacokinetic analysis. $J$ Clin Pharm Ther, 36(5): 614-624.

Silverman JA. 1999. Multidrug-resistance transporters. Pharm Biotechnol, 12: 353386.

Sparreboom A, van Asperen J, Mayer U, Schinkel AH, Smit JW, Meijer DK, Borst P, Nooijen WJ, Beijnen JH, van Tellingen O. 1997. Limited oral bioavailability and active epithelial excretion of paclitaxel (Taxol) caused by P-glycoprotein in the intestine. Proc Natl Acad Sci USA, 94(5): 2031-2035.

Stearns RA, Chakravarty PK, Chen R, Chiu SH. 1995. Biotransformation of losartan to its active carboxylic acid metabolite in human liver microsomes. Role of cytochrome P4502C and 3A subfamily members. Drug Metab Dispos, 23(2): 207-215.

Sujatha M, Sukesan S, Kiran U. 2011. Can the use of sevoflurane with rocuronium reconcite the debate on succinylcholine versus rocuronium for rapid sequence 
intubation? Korean J Anasthesiol, 61(1): 159-161.

Tang K, Ngoi SM, Gwee PC, Chua JM, Lee EJ, Chong SS, Lee CG. 2002. Distinct haplotype profiles and strong linkage disequilibrium at the MDR1 multidrug transporter gene locus in three ethnic Asian populations. Pharmacogenetics, 12(6): 437-450.

Valente RC, Capella LS, Nascimento CR, Braga F, Echevarria-Lima J, Lopes AG, Capella MA . 2008. ABCB1 (Pglycoprotein) but not ABCC1 (MRP1) is downregulated in peripheral blood mononuclear cells of spontaneously hypertensive rats. Pflugers Arch, 456(2): 359-368.

Verschraagen M, Koks CH, Schellens JH, Beijnen JH. 1999. P-glycoprotein system as a determinant of drug interactions: the case of digoxin-verapamil. Pharmacol Res, 40(4): 301-306.

Washington CB, Duran GE, Man MC, Sikic BI, Blaschke TF. 1998. Interaction of anti-HIV protease inhibitors with the multidrug transporter P-glycoprotein (Pgp) in human cultured cells. J Acquir Immune Defic Syndr Hum Retrovirol, 19(3): 203-209.

Yanagimachi M, Naruto T, Tanoshima R, Kato H, Yokosuka T, Kajiwara R, Fujii H, Tanaka F, Goto H, Yagihashi T, Kosaki K, Yokota S. 2010. Influence of CYP3A5 and ABCB1 gene polymorphisms on calcineurin inhibitorrelated neurotoxicity after hematopoietic stem cell transplantation. Clin Transplant, 24(6): 855-861. 